\title{
Study on LCL type IPT system power bi-directional push mode
}

\author{
Jian $\mathrm{Xu}^{1, *}$, Yongsheng Zhang ${ }^{2}$, and Lei Gan ${ }^{1}$ \\ ${ }^{1}$ Institute of Mechanical Manufacturing Technology, China Academy of Engineering Physics,621900 Mianyang China \\ ${ }^{2}$ Department of Mechanical Engineering Sichuan Engineering Technical College ,618000 Deyang China
}

\begin{abstract}
Aiming at the working principle of inductive power transfer (IPT) technology, a bi-directional power conversion topology based on LCL type composite resonance is proposed. This topology introduces the advantages of LCL type resonance into the bi-directional power topology which has a resonance capacity larger than the single stage LC, it has a strong load adaptability, a smaller switching device voltage and current stress. Aiming at the LCL bi-directional power topology proposed, its working principle is introduced in detail, and the AC impedance model and mathematical model of the system are established. On this basis, the feasibility is verified by using the simulation experiment results.
\end{abstract}

\section{Introduction}

Inductive power transfer technology, as a brand-new research field, has received extensive attention in recent years and it is widely used in many fields like electric vehicle power supply, special environment of underwater mine. However, due to the one-way transmission characteristic of conventional IPT, a series of problems exist in the application of high-power types (materials handling equipment, urban electrified transportation etc.) such as power traction. These include the difficulty of electric power feedback resulted by the one-way transmission characteristic and a lower power transmission efficiency resulted by the open loop of electric power transmission.

In order to solve the problems above, a bi-directional high frequency electric power push mode is proposed in document [1], which is used to realize the bi-directional transmission of power electric transfer between the transmitting end and the receiving end. The characteristics of the mode lies in: The transmitting and receiving end of the system both use peer-to-peer topology, which works in magnetic field excitation and receiving mode to satisfy the bi-directional transmission of energy. Meanwhile, according to the working state and need, the direction and size of power electric push can be changed dynamically, and then the operation efficiency of the system is increased.

The bi-directional electric energy topology proposed in document [2] is a symmetrical SS type. The topology has a characteristic: the current in the resonance coil all passes through the high-frequency inverter circuit or high frequency rectifier circuit, so the switching device bears rather big current stress. Especially in high-power application, the heating problem of the switching device is very serious. To deal with this problem, bi-directional power conversion topology based on LCL type composite resonance is proposed. This topology introduces the advantages of LCL type resonance into the bi-directional power topology. It has characteristics as follows: a larger resonance capacity than single-level LC resonance; a strong load adaptability with lower resonance frequency drift; a lower voltage and current stress of switching device at the same power level.

Aiming at the LCL bi-directional power topology proposed, its working principle is introduced in detail, and the AC impedance model and mathematical model of the system are established. On this basis, the feasibility is verified by using the simulation experiment results.

\section{The working principle of LCL type bi- directional power conversion topology}

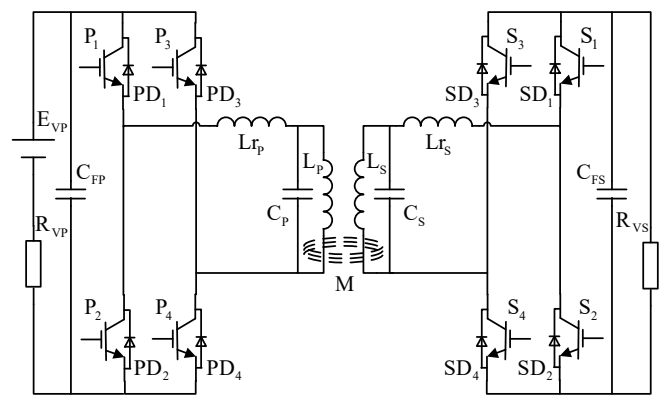

Fig.1 LCL type bi-directional high frequency AC power conversion topology

Fig. 1 is the LCL type bi-directional high frequency AC power topology. Taking the $\mathrm{P}$ end topology as an example, its high frequency conversion is formed by full-bridge circuit which consists of four switch tubes

(P1 P4) and anti-parallel diode (PD1 PD4) .The full-bridge circuit can operate in rectification and inverter modes. LrP, CP and LP form the LCL type

\footnotetext{
* Corresponding author: 276558268@qq.com
} 
composite resonance network, which is used to generate high frequency resonance in order that the $\mathrm{S}$ end receives magnetic field energy.

Document [3] has proposed that without extra adding power conditioning link, dynamical adjustment of power transmission can be realized and the system can operate in energy injection mode and energy free oscillation mode. Taking the $\mathrm{P}$ end as the energy transmitting end and $\mathrm{S}$ end as the receiving end as an example to illustrate. They are shown in Fig.2.

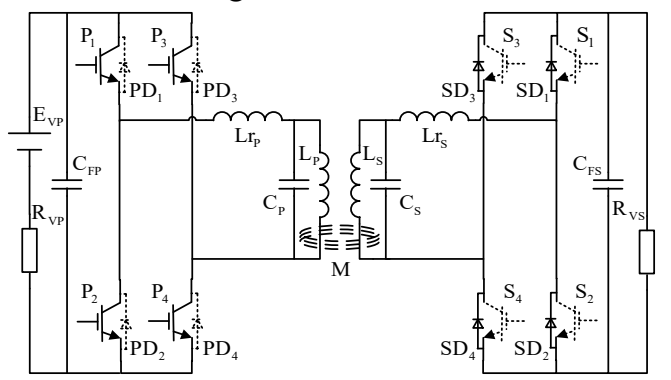

(a) Working mode 1 (energy injection working mode)

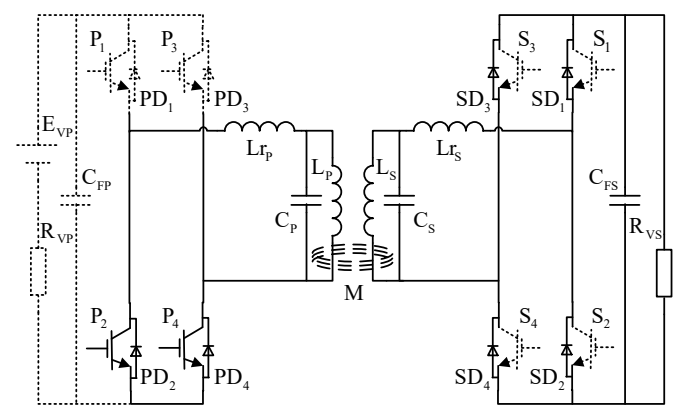

(b) Working mode 2 (energy free oscillation mode)

Fig .2 dual-working mode structure diagram

Working mode 1 is the energy injection mode, where (P1, P4) and (P2, P3) alternatively conduct and convert the DC input EVP into high frequency square wave voltage, which is injected into the resonant network made up of LrP, CP and LP. So, high frequency sine wave energy output is generated in transmit coil. By detecting the current in the resonant inductor $\mathrm{Lr}$ and controlling the alternative conduction of two sets of switch tubes (P1, P4) and (P2, P3), the switch of switch tubes can always conduct at the zero-crossing point of resonant current, where the loss of switch tubes is zero. Then, the operation of ZCS (zero current switching) is realized.

Working mode 2 is the energy free oscillation mode, where ( P2, PD4) and ( P4, PD2) form the bidirectional freewheel switch pair to maintain the bidirectional energy flow of resonant link. In this case, the systems completely rely on energy storage of resonant link to maintain autonomous oscillation. Similarly, these two pairs of switch tubes can detect the zero-crossing point of resonant current to realize the operation of ZCS.

By dynamically controlling two working modes of energy emission, dynamic adjustment of input power and output voltage can be realized. In these two modes, the $\mathrm{S}$ end is always in energy receiving state. ( $\mathrm{S} 1, \mathrm{~S} 2$, S3, S4) all turn off. (SD1, SD4) and (SD2, SD3 ) form the full-bridge rectifier circuit, which completes the bi-directional rectifier of high frequency sine wave and output to load RVS.

\section{AC Impedance analysis}

As the LCL type bi-directional power conversion topology is a symmetrical structure, so the circuit parameter of $\mathrm{P}$ end and Send must be the same, which means the parameters of resonant inductors $\left(L r_{P}, L r_{S}\right)$ and $\left(L_{P}, L_{S}\right)$ and that of resonant capacitance should be consistent. The following assumes the power push direction is from $\mathrm{P}$ end (transmitting end) to $\mathrm{S}$ end (receiving end).

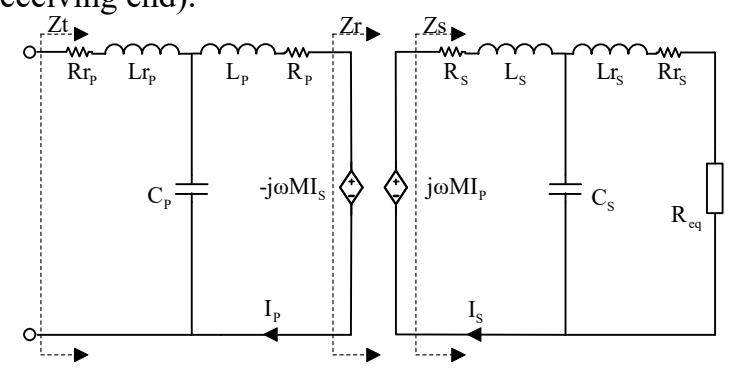

Fig.3 equivalent electrical circuit of main circuit

The AC impedance model of system resonant circuit is shown in Fig.3, where $-j \omega M I_{S}$ and $j \omega M I_{P}$ are mutual inductance electromotive force of $\mathrm{P}$ end and $\mathrm{S}$ end. The rectifier link, filtering link and load can be equivalent as $R_{e q}$.

The total impedance $Z_{S}$ of secondary side circuit is:

$$
\begin{aligned}
Z_{S}= & \frac{j \omega M I_{P}}{I_{S}}=j \omega L_{S}+R_{S} \\
& +\frac{1}{j \omega C_{S}+1 /\left(j \omega L r_{S}+R r_{S}+R_{e q}\right)}
\end{aligned}
$$

The reflection impedance $Z_{r}$ equivalent to $\mathrm{P}$ end equals the reflection electromotive force $-j \omega M I_{S}$ divided by the current $I_{P}$ :

$$
Z_{r}=\frac{-j \omega M I_{S}}{I_{P}}=\frac{\omega^{2} M^{2}}{Z_{S}}
$$

The total impedance of system $Z_{t}$ is:

$$
\begin{aligned}
Z_{t}= & j \omega L r_{P}+R r_{P} \\
& +\frac{1}{j \omega C_{P}+1 /\left(j \omega L_{P}+R_{P}+Z_{r}\right)}
\end{aligned}
$$

When system is in complete resonance, the imaginary part of total impedance is $Z_{t}$ zero and the phase angle is zero. That is : 


$$
\operatorname{Im}\left(Z_{t}\right)=0 \text { or } \angle Z_{t}=0
$$

Inherent resonance frequency (zero phase angles) of system can be solved by combining formulas (1) to (4).

\section{LCL type bi-directional high frequency AC power conversion mathematical model}

In order to analyze the topology of LCL type directional high frequency $\mathrm{AC}$ power conversion, mathematical models needs to be established for two working modes. Aiming at the working mode 1 shown in Fig.2 (a), its correspondent equivalent circuit model can be established, shown in Fig.4.

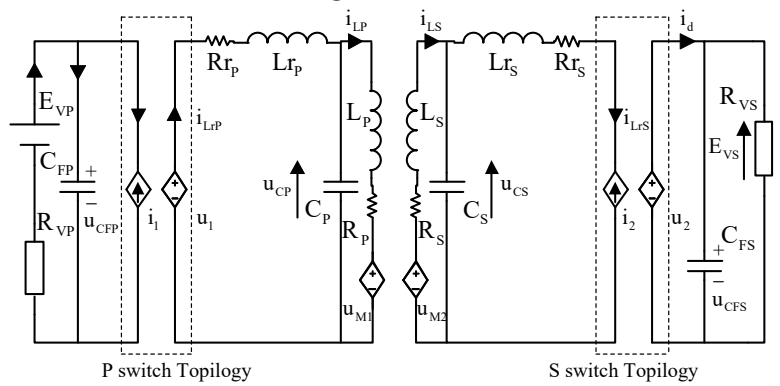

Fig.4 Equivalent circuit diagram of working mode 1

The switch topology of $\mathrm{P}$ end can be equivalent as current-controlled voltage source, where input current $i_{1}=i_{L r P} S(t)$ so the voltage of the controlled voltage source $u_{1}=u_{C F P} S(t) \quad$ Where, $S(t)$ is a non-linear switching function. Assume that $t_{i}\left(i \in Z^{+}\right)$is the switching moment of ZVS switch then $S(t)$ can be expressed as follows :

$$
S(t)= \begin{cases}1, & t \leq t_{i} \\ -1, & t>t_{i}\end{cases}
$$

The switch topology of $\mathrm{S}$ end can also be equivalent as current-controlled voltage source, where the input current $i_{2}=i_{d} \operatorname{sgn}\left(i_{L r S}\right)$ so the controlled voltage source $u_{2}=u_{C F S} \operatorname{sgn}\left(i_{L r S}\right)$. Where $\operatorname{sgn}\left(i_{L r S}\right)$ is sign function, expressed as follows:

$$
\operatorname{sgn}\left(i_{L r S}\right)= \begin{cases}1, & i_{L r S}>0 \\ 0, & i_{L r S}=0 \\ -1, & i_{L r S}<0\end{cases}
$$

According to formula (5) and (6), the following eighth order differential equation can be established:

$$
\left\{\begin{aligned}
u_{C F P}^{\prime}= & C_{F P}^{-1} R_{V P}^{-1} E_{V P}-C_{F P}^{-1} R_{V P}^{-1} u_{C F P}+C_{F P}^{-1} S(t) i_{L r P} \\
i_{L r P}^{\prime}= & L r_{P}^{-1} S(t) u_{C F P}-R r_{P} L r_{P}^{-1} i_{L r P}-L r_{P}^{-1} u_{C P} \\
u_{C P}^{\prime}= & C_{P}^{-1} i_{L r P}-C_{P}^{-1} i_{L P} \\
i_{L P}^{\prime}= & -\Delta^{-1} L_{S} u_{C P}+\Delta^{-1} L_{S} R_{P} i_{L P} \\
& +\Delta^{-1} M u_{C S}+\Delta^{-1} M R_{S} i_{L S} \\
i_{L S}^{\prime}= & -\Delta^{-1} M u_{C P}+\Delta^{-1} M R_{P} i_{L P} \\
& +\Delta^{-1} L_{P} u_{C S}+\Delta^{-1} L_{P} R_{S} i_{L S} \\
u_{C S}^{\prime}= & C_{S}^{-1} i_{L S}-C_{S}^{-1} i_{L r S} \\
i_{L r S}^{\prime}= & L r_{S}^{-1} u_{C S}-R r_{S} L r_{S}^{-1} i_{L r S} \\
& -L r_{S}^{-1} u_{C F S} \operatorname{sgn}\left(i_{L r S}\right) \\
u_{C F S}^{\prime}= & C_{F S}^{-1} i_{L r S} \operatorname{sgn}\left(i_{L r S}\right)-C_{F S}^{-1} R_{V S}^{-1} E_{V S}
\end{aligned}\right.
$$

Where, $\Delta=M^{2}-L_{P} L_{S}$

For working mode 2 shown in Fig. 2(b), the correspondent equivalent circuit model can be established, shown in Fig.5:

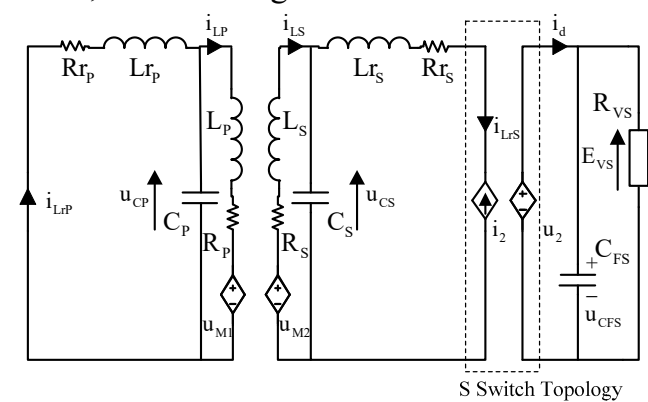

Fig. 5 equivalent circuit diagram of working mode 2

According to the equivalent circuit model, the seventh differential equation can be expressed as:

$$
\left\{\begin{aligned}
i_{L r P}^{\prime} & =-R r_{P} L r_{P}^{-1} i_{L r P}-L r_{P}^{-1} u_{C P} \\
u_{C P}^{\prime} & =C_{P}^{-1} i_{L r P}-C_{P}^{-1} i_{L P} \\
i_{L P}^{\prime} & =-\Delta^{-1} L_{S} u_{C P}+\Delta^{-1} L_{S} R_{P} i_{L P}+\Delta^{-1} M u_{C S}+\Delta^{-1} M R_{S} i_{L S} \\
i_{L S}^{\prime} & =-\Delta^{-1} M u_{C P}+\Delta^{-1} M R_{P} i_{L P}+\Delta^{-1} L_{P} u_{C S}+\Delta^{-1} L_{P} R_{S} i_{L S} \\
u_{C S}^{\prime} & =C_{S}^{-1} i_{L S}-C_{S}^{-1} i_{L r S} \\
i_{L r S}^{\prime} & =L r_{S}^{-1} u_{C S}-R r_{S} L r_{S}^{-1} i_{L r S}-L r_{S}^{-1} u_{C F S} \operatorname{sgn}\left(i_{L r S}\right) \\
u_{C F S}^{\prime} & =C_{F S}^{-1} i_{L r S} \operatorname{sgn}\left(i_{L r S}\right)-C_{F S}^{-1} R_{V S}^{-1} E_{V S}
\end{aligned}\right.
$$

Where, $\Delta=M^{2}-L_{P} L_{S}$

In these two working modes, according to the different value of $S(t)$ and $\operatorname{sgn}\left(i_{L r S}\right)$, four operating modes can be obtained. Letter $j$ stands for operating mode and letter $i$ stands for mode indicators of given modes. State space equation can be established as : $\dot{x}_{j}=A_{j i} x_{j}+B_{j i} E, \quad i=1,2,3,4, j=1,2$

Where,

$$
\begin{aligned}
& x_{1}=\left[u_{C F P}, i_{L r P}, u_{C P}, i_{L P}, i_{L S}, u_{C S}, i_{L r S}, u_{C F S}\right]^{\mathrm{T}} ; \\
& x_{2}=\left[i_{L r P}, u_{C P}, i_{L P}, i_{L S}, u_{C S}, i_{L r S}, u_{C F S}\right]^{\mathrm{T}} ; \\
& E=\left[E_{V P}, E_{V S}\right]^{\mathrm{T}}
\end{aligned}
$$




\section{Simulation analysis}

In order to verify the effectiveness of LCL type bidirectional high frequency $\mathrm{AC}$ power conversion topology and its mathematical model, Matlab environment is based to analyze and the parameters used are shown in Tab. 1

Table 1. Simulation Parameters

\begin{tabular}{|c|c|c|c|}
\hline $\begin{array}{c}\text { P end } \\
\text { Parameters }\end{array}$ & Value & $\begin{array}{c}\text { S end } \\
\text { Parameters }\end{array}$ & Value \\
\hline$L r_{P} / \mu H$ & 869 & $L r_{S} / \mu H$ & 869 \\
\hline$L_{P} / \mu H$ & 400 & $L_{S} / \mu H$ & 400 \\
\hline$C_{P} / \mu F$ & 0.15 & $C_{S} / \mu F$ & $0.15 \mathrm{~s}$ \\
\hline$M / \mu H$ & 40 & $R_{V S} / \Omega$ & 200 \\
\hline
\end{tabular}

The power push mode is designed to push from $\mathrm{P}$ end to $\mathrm{S}$ end (forward push). The time of system simulation is set as $0 \sim 60 \mathrm{~ms}$. When the system enters a steady state, waveforms of $\mathrm{P}$ end and $\mathrm{S}$ end resonant capacitance voltage (30ms 30.1ms) are shown in Fig.6.

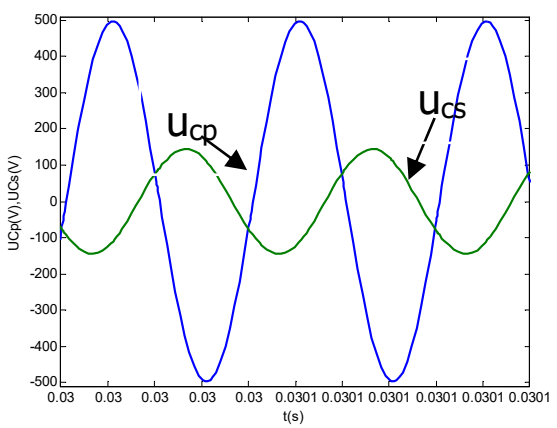

Fig. $6\left(u_{C P}, u_{C S}\right)$ Voltage waveforms of the $\mathrm{P}$ end and $\mathrm{S}$ end

It can be seen from Fig.6 that both resonant voltage waveforms of $\mathrm{P}$ end and $\mathrm{S}$ end are sinusoidal waveforms with lower distortions.

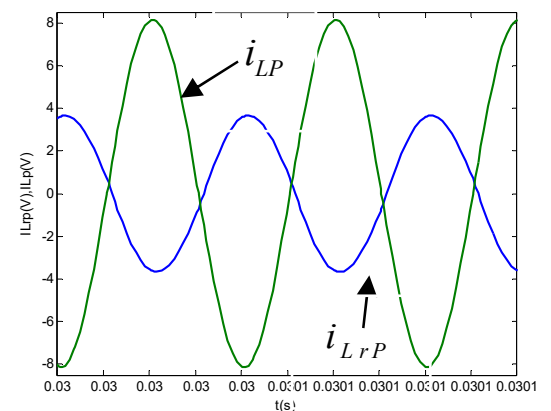

Fig. $7\left(i_{L r P}, i_{L P}\right)$, resonant current waveforms of the $\mathrm{P}$ end shows the current waveform of resonant

$L r$ and $L_{P}$ of the P end. It can be seen that the peak value of $i_{L r P}$ is approximately half that of $i_{L P}$. So , compared with the single-level LC resonance, under the same transmission power, stress in the switch tube is obviously lower.

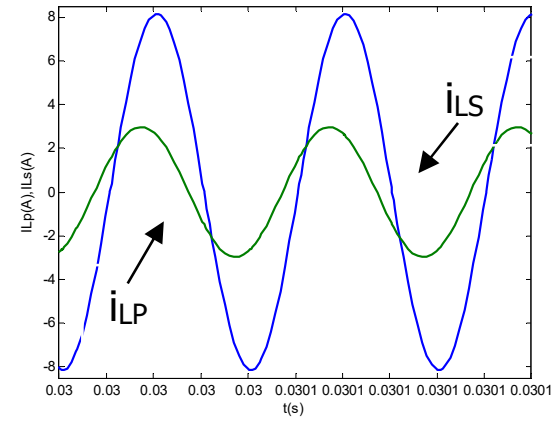

Fig. $8\left(i_{L P}, i_{L S}\right)$, resonant current waveforms of $\mathrm{P}$ end and $S$ end

Fig. 8 shows the current waveform of resonant capacitance $L_{P}$ and $L_{S}$ of the $\mathrm{P}$ end. It can be seen that $i_{L P}$ and $i_{L S}$ are also sinusoidal waveforms with lower distortions.

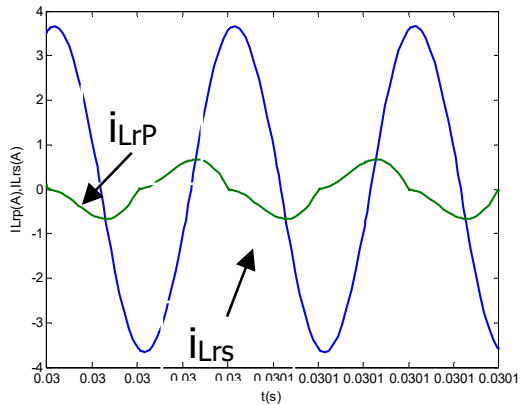

Fig. $9\left(i_{L r P}, i_{L r S}\right)$, resonant current waveforms of $\mathrm{P}$ end and $\mathrm{S}$ end

Fig. 9 shows the current waveform of resonant capacitance $L r_{P}$ and $L r_{S}$ of the P end. It can be seen that $i_{L r P}$ is a sinusoidal waveform with lower distortion while that of $i_{L r S}$ is larger, caused by the filtering capacitance $C_{F S}$

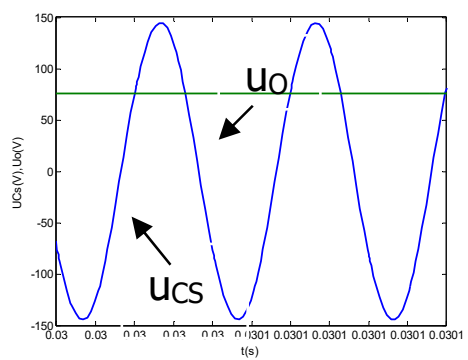

Fig.10 $\left(u_{C S}, u_{o}\right)$, resonant and output voltage waveforms of $\mathrm{S}$

Fig. 10 shows the resonant capacitance voltage $u_{C S}$ of the $\mathrm{S}$ end and the load output voltage $u_{o}$.It can be seen that, with energy injection and free oscillation modes alternatively operate, output voltage $u_{o}$ keeps constant well at $75 \mathrm{~V}$. As the structure of the $\mathrm{P}$ end and $\mathrm{S}$ end is symmetrical, backward and forward push modes are the same, which is not described further.

The above simulation results show that, for LCL type 
bi-directional power push topology, whether forward push or backward push, working modes switch can keep a constant output voltage. The generated waveform distortion of the resonant voltage and current are in an acceptable range, which is beneficial for improving energy push efficiency and reducing the EMI disturbance to the environment.

\section{Conclusion}

It can be seen from simulation results that, the proposed LCL type power bi-directional push topology successfully overcomes the weaknesses of the singlelevel LC type bi-directional power conversion topology device, where it bears a larger electronic stress. The bidirectional push of energy is also realized. For LCL type bi-directional circuit topology proposed, working methods are introduced in detail. Meanwhile, the AC impedance model and mathematical model are established. On this basis, the feasibility is verified by using simulation.

\section{References}

1. ZHANG Chun-lin, ZHANG Dong-hui, YAN Ping .Analysis and Study on LCL Resonant Converter[J] High Voltage Apparatus 2007,43(5)

2. MA Gang, QU Wen-long, LIU Yuan-yuan Isolated soft switching bidirectional DC/DC converter[J], Tsinghua Univ (Sci \&Tech) 2006,46(10).

3. MA Gang, QU Wen-long, LIU Yuan-yuan, Steady State Analysis of a Bidirectional DC/DC Converter, Power Electronics, 2007,41(5).

4. MA Gang, QU Wen-long, LIU Yuan-yuan, A Novel Soft Switching Bidirectional DC/DC Converter and Its ZVS Condition ,TRANSACTIONS OF CHINA ELECTROTECHNICAL SOCIETY,2006,21(7).

5. Wu Jun-hui, Huang Sheng-hua, Zou Jinag-feng, Three-Level Bidirectional DC/DC Converters For Hybrid Electrical Vehicle. Drive \& Control, 2006(2).

6. Yugang Su Chunsen Tang Shuping Wu, ,Research of LCL Resonant Inverter In Wireless Power Transfer System, Power System Technology,2006

7. Duleepa J.T,Udaya K .M,a Novel Matrix Converter Based Bi-Directional IPT Power Interface for V2G Applications, IEEE International Energy Conference , 2010 